\title{
Produção científica da Terapia Ocupacional na inclusão escolar: interface com a Educação Especial e contribuições para o campo
}

\author{
Aline Cirelli Coppede* \\ Alyne Kalyane Câmara de Oliveira** \\ Fernanda Duarte Rosa*** \\ Maria Cristina Piumbato Innocentini Hayashi****
}

\section{Resumo}

Uma estratégia que tem se mostrado produtiva para que a educação inclusiva seja posta em prática de forma eficaz é a parceria de profissionais da educação com profissionais da saúde. O terapeuta ocupacional (TO) apresenta em sua formação, características capazes de enquadrá-lo como elemento importante no processo de melhorias na inclusão escolar. Objetiva-se caracterizar a produção científica nacional acerca das contribuições da Terapia Ocupacional no processo de inclusão escolar, através de uma pesquisa exploratória e descritiva, com abordagem bibliométrica. As buscas foram realizadas nas bases de dados da BVS, Scielo, Redalyc e nos periódicos Cadernos de Terapia Ocupacional da UFSCar, Revista de Terapia Ocupacional da USP, Revista Brasileira de Educação Especial e Revista Educação Especial da UFSM, utilizando as expressões de busca "terapia ocupacional", "inclusão escolar", "educação especial", "educação inclusiva", "contexto escolar", "tecnologia assistiva" e "escola". Com o auxílio do Software VantagePoint ${ }^{\circledR}$, as análises produziram indicadores: ano de publicação; principais periódicos nos quais estes artigos são publicados; autores que mais participam de publicações; principais temas abordados. Resultados apontaram algumas características da literatura da área, com destaque para o crescimento no número de publicações nos últimos dois anos; predominância de publicações em periódicos da Terapia Ocupacional; pequeno grupo de autores responsáveis pela maioria das publicações; maior parte de estudos originais, de revisão, exploratórios e descritivos; sendo a temática mais frequente referente a questões sobre a atuação do TO em processos de inclusão escolar, dados estes que caracterizam a produção científica e revelam importantes contribuições da Terapia Ocupacional no contexto da educação inclusiva.

Palavras-chave: Educação Especial; Terapia Ocupacional; Inclusão escolar.

\footnotetext{
* Doutoranda no Programa de Pós-Graduação em Educação Especial da Universidade Federal de São Carlos, São Carlos, São Paulo, Brasil.

** Doutoranda do Programa de Pós-Graduação em Educação Especial da Universidade Federal de São Carlos, São Carlos, São Paulo, Brasil.

*** Doutoranda do Programa de Pós-Graduação em Educação Especial da Universidade Federal de São Carlos, São Carlos, São Paulo, Brasil

**** Professora Doutora da Universidade Federal de São Carlos (UFSCar), São Carlos, São Paulo, Brasil.
} 


\section{Scientific Production of occupational therapy in the school inclusion: interface with the Special Education and contributions to the field}

\section{Abstract}

A strategy, which has been proved productive to inclusive education, was put into practice, in an effective way, by the education and health professionals partnership. The occupational therapist (OT) presents, in its formation, characteristics which enable them to frame as important elements in the process of improvement in the school inclusion. It aims to characterize the national scientific production about occupational therapy contributions for school inclusion process, through an exploratory and descriptive search, with a bibliometric approach. The search was performed in the databases BVS, Scielo, Redalyc and periodics Cadernos de Terapia Ocupacional da UFSCar, Revista de Terapia Ocupacional da USP, Revista Brasileira de Educação Especial e Revista Educação Especial da UFSM, using the search expressions "occupational therapy", "school inclusion", "especial education", "inclusive education" "school context", "assistive technology" and "school". With the aid of software VantagePoint ${ }^{\circledR}$, the analysis produced indicators: year of publication, major journals when these papers were published, authors who participated in more publications, main topics discuted. Results pointed some characteristics of the literature of the field, highlighting the growing number of publishing in the last two years; publication predominance in occupational therapy journals; small group of authors responsible for the majority of publications, most studies are original, review, exploratory and descriptive, being the most common theme regarding questions about the role of OT in school inclusion processes, these data that characterize the scientific and reveal important contributions of occupational therapy in the context of inclusive education.

Keywords: Special Education; Occupational Therapy; School inclusion.

\section{Introdução}

O processo de inclusão escolar ainda apresenta muitas demandas para que os serviços educacionais possam oferecer ações efetivas que promovam igualdade de oportunidades e inclusão. Dentre as necessidades apontadas pelos educadores, encontram-se a falta de um maior número de profissionais especializados para o oferecimento de suporte aos professores e a falta de parcerias efetivas com serviços de saúde (IDE, YAMAMOTO; SILVA, 2011).

O terapeuta ocupacional (TO), assim como demais profissionais da saúde, apresenta, em sua formação, características que o tornam apto para contribuir no contexto da educação inclusiva. Por se dedicar ao estudo do desenvolvimento humano e ao processo de aprendizagem e autonomia, a Terapia Ocupacional, enquanto ciência e profissão apresenta arcabouço teórico e prático para contribuir, inclusive, 
com a desmistificação da abordagem à pessoa com necessidades educacionais especiais (MUNGUBA, 2007).

Segundo Rocha, Luiz e Zulian (2003), a inserção do terapeuta ocupacional, em contextos escolares, não é clínica, não é voltada a demandas específicas de alunos com deficiência, e não é direcionada a rever questões pedagógicas, mas sim um trabalho a ser desenvolvido em conjunto com os educadores, alunos, pais e comunidade; que possa oferecer oportunidade para que as reais dificuldades e emoções presentes, nesse processo, possam ser reveladas, e, a partir destas, possam ser propostas estratégias para superar barreiras, fortalecendo as potencialidades de educadores e educandos.

Iniciativas e projetos têm apresentado diversas possibilidades de ação para o terapeuta ocupacional dentro do contexto da educação inclusiva, como o oferecimento de suporte técnico e emocional a professores e alunos, ações de consultoria colaborativa, confecção de adaptações e auxílio na elaboração de atividades (TOYODA et al., 2007). Esse profissional também pode contribuir facilitando o acesso de alunos com deficiência, ao participar da adequação do ambiente físico das escolas, adaptando mobiliários, recursos de informática, programas e equipamentos. O terapeuta ocupacional pode atuar na introdução de tecnologia assistiva adequada, comunicação alternativa e realizar encaminhamentos para serviços de saúde e de reabilitação, quando necessário (ROCHA, LUIZ; ZULIAN, 2003).

Estudos apontam a importância do terapeuta ocupacional em ações de implementação e adaptação de tecnologias assistivas (PELOSI, 2005; PELOSI e NUNES, 2011), e que a participação desse profissional em trabalhos de parceria colaborativa com educadores tem se mostrado bastante positiva (TREVISAN; DELLA BARBA, 2012).

Contudo, estudos também assinalam a existência de dificuldades na inserção do terapeuta ocupacional em contextos escolares, como dificuldades de escopo político, que ainda deixam em aberto a questão sobre a forma como esse profissional pode atuar na inclusão escolar (TOYODA et al., 2007), a falta de conhecimento do papel deste profissional entre os profissionais da educação (IDE, YAMAMOTO; SILVA, 2011) e a necessidade de uma maior instrumentalização e qualificação profissional nos cursos de formação em Terapia Ocupacional, para atuar de maneira mais satisfatória nos processos de inclusão escolar (ANDRADE; BRAGA, 2009).

Diante dessas constatações apontadas pela literatura, o presente texto apresenta os resultados de um estudo com abordagem bibliométrica, que teve como objetivo caracterizar a produção científica nacional acerca das contribuições da Terapia Ocupacional no processo de inclusão escolar. Estudos dessa natureza são importantes porque oferecem subsídios aos pesquisadores para avaliar e questionar os avanços na área ao longo do tempo, além de apontar as perspectivas e tendências da área, identificando as lacunas existentes cuja solução depende da investigação científica e de intervenções que estejam embasadas cientificamente (PINHEIRO et al., 2011). 


\section{Método}

Trata-se de uma pesquisa de caráter exploratório e descritivo, com abordagem bibliométrica. Segundo Pizanni, Silva e Hayashi (2008), a bibliometria é uma ferramenta que permite observar o estado da ciência e da tecnologia através da produção da literatura científica como um todo, em um determinado nível de especialização. Por meio da bibliometria é possível construir indicadores destinados a avaliar a produção científica de indivíduos, áreas de conhecimento e países, sendo estes indicadores empregados na avaliação de pesquisadores e áreas de conhecimento (SILVA, HAYASHI; HAYASHI, 2011).

Para coleta de dados, foram selecionados artigos presentes nas seguintes bases de dados: Biblioteca Virtual em Saúde (BVS), Scientific Eletronic Library Online (Scielo), Rede de Revistas Científicas para América Latina, Caribe, Espanha e Portugal (Redalyc) e os periódicos Cadernos de Terapia Ocupacional da UFSCar, Revista de Terapia Ocupacional da USP, Revista Brasileira de Educação Especial e Revista Educação Especial, todos disponíveis online, através das expressões de busca: "terapia ocupacional", "inclusão escolar", "educação especial", "educação inclusiva", "contexto escolar", "tecnologia assistiva" e "escola". Os dados foram coletados em 15/07/2012.

As bases foram escolhidas devido a sua abrangência e por serem importantes fontes de informação em pesquisas na interface educação e saúde, área esta que se concentra a temática do estudo em questão. E os periódicos por serem os mais específicos da temática abordada: Educação Especial e Terapia Ocupacional. Após a definição dos termos, foram acessadas as bases de dados e os periódicos selecionados e realizou-se a busca em cada base/periódico separadamente. Não foi delimitado o período de busca, pois o interesse consistiu em mapear a evolução da produção científica nacional sobre o tema investigado. Assim, foi considerado desde o registro mais antigo até o mais atual.

Considerou-se como critério de inclusão apenas os artigos que traziam a expressão "terapia ocupacional" e/ou "inclusão escolar" no título, resumo ou palavrachave. Foram excluídos os artigos que tratavam somente de Terapia Ocupacional, sem fazer relação desta com a inclusão escolar, ou ainda artigos que abordavam a inclusão escolar, mas que não discutiam o papel da Terapia Ocupacional. Além disso, foram excluídos os resumos que estavam incompletos ou que não foram possíveis de acessar o texto na íntegra, e os estudos que se repetiram em mais de uma base/periódico foram considerados apenas uma vez.

Com a amostra final dos artigos selecionados, os quais contemplaram todos os critérios de inclusão previamente delimitados, os dados coletados foram registrados e organizados em um protocolo de dados bibliométricos elaborado por Hayashi et al. (2011), no qual foram registrados os seguintes aspectos: base de dados; periódico; ano de publicação; delineamento da pesquisa; tipologia dos estudos; amostra/participantes; instrumento para coleta de dados; e principais temáticas relacionadas ao assunto do estudo. Em seguida, foi realizada uma leitura minuciosa de todos os registros e, posteriormente, os dados foram transpostos para o software VantagePoint ${ }^{\circledR}$, que permite realizar as análises bibliométricas. Os indicadores bibliométricos resultantes 
foram formatados em tabelas e gráficos com o auxílio do software MS Excel, de forma a propiciar maior visibilidade dos resultados alcançados.

Quanto aos aspectos éticos, como se trata de uma pesquisa documental, dispensa-se a exigência de submissão e aprovação do Comitê de Ética. Os dados coletados e analisados, bem como os nomes de seus autores, são de domínio público, disponíveis em bases de dados online. Nesse contexto, os aspectos éticos da pesquisa científica referem-se à honestidade e precisão com relação aos dados coletados, o que implicou em respeito à autoria científica e fidedignidade às ideias dos autores analisados.

\section{Resultados e discussão}

A princípio, apresentam-se detalhadamente os resultados dos artigos selecionados em cada base e periódico investigado.

Na BVS, utilizou-se a expressão "terapia ocupacional" e idioma "português", resultando em 511 artigos. Refinando, no assunto principal para "pessoas com deficiência" e "crianças com deficiências", obtiveram-se 18 e 15 artigos respectivamente, dos quais 3 atendiam aos critérios de inclusão. Na Scielo, utilizou-se a expressão "terapia ocupacional" que resultou em 35 artigos, dos quais 3 foram selecionados. Com o termo "educação inclusiva/terapia" resultou em 2 artigos, porém estes eram repetidos. E na Redalyc, utilizou-se a expressão "terapia ocupacional/educação especial", que resultou em 1 artigo considerado pertinente para a pesquisa.

Já, nos periódicos investigados, utilizou-se para a busca na Revista de Terapia Ocupacional da USP as expressões "educação especial", "inclusão escolar" e "escola", resultando em 34, 3 e 2 artigos, respectivamente, sendo selecionados desse total apenas 7 artigos. Na revista Cadernos de Terapia Ocupacional da UFSCar foram utilizadas as expressões "educação especial", "educação inclusiva", "inclusão escolar", "contexto escolar" e "tecnologia assistiva", sendo recuperados 5, 7, 8, 7 e 5 artigos, respectivamente. Destes, 14 foram selecionados, 3 foram excluídos e 15 eram repetidos. Por sua vez, na Revista Brasileira de Educação Especial e na Revista Educação Especial, a expressão "terapia ocupacional" resultou em 2 e 3 artigos respectivamente, sendo todos selecionados para a pesquisa. No total, foram eleitos 33 artigos, cujas temáticas se relacionavam à participação direta ou indireta do terapeuta ocupacional em contextos de inclusão escolar.

Ao analisar a produção científica, ao longo dos anos, foi possível observar a evolução e crescimento deste campo de conhecimento. A Figura 1 apresenta a distribuição das publicações ao longo dos anos. 


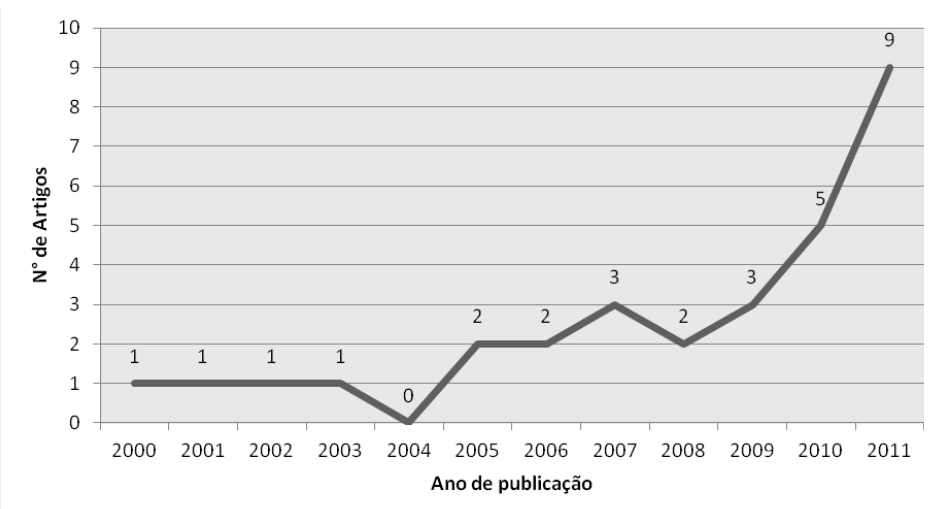

Figura 1. Distribuição dos artigos recuperados ao longo dos anos.

Os dados mostram uma distribuição uniforme até 2003, não sendo registrado nenhum artigo em 2004. Entre 2005 e 2009 há um discreto aumento das publicações, com destaque para crescimento nos dois últimos anos, sendo 2010 com 5 artigos e 2011 com 9 artigos. Assim, a contribuição científica da Terapia Ocupacional, na inclusão escolar, tem mostrado um crescimento ao longo dos anos, podendo-se inferir que o interesse por essa temática na comunidade científica tem aumentado. Além disso, outro aspecto a ser considerado em relação ao interesse da comunidade científica pelo tema da inserção do terapeuta ocupacional, no contexto escolar inclusivo, pode ser buscado na constatação de que a partir de 2005, de acordo com dados do Censo Escolar de 2005 (MEC/INEP). Houve crescimento no atendimento inclusivo no país, bem como aumentou o número de crianças com necessidades especiais matriculadas na rede pública de ensino. Com isso, o papel dos diversos profissionais envolvidos no processo de inclusão escolar - entre eles, o terapeuta ocupacional - passou a ser investigado pela comunidade científica, refletindo em crescimento da literatura.

De acordo com Laudelino, Navarro e Beuren (2010), estudar a evolução das pesquisas, ao longo do tempo, é um fator importante, pois a contagem das publicações, como indicador bibliométrico, é um instrumento de análise que se presta a difundir achados científicos e colabora com a política de revisão científica destinada a medir e avaliar as contribuições à ciência, demonstrando o fortalecimento ou o aumento do interesse de um determinado conhecimento pela comunidade científica.

Com relação à frequência de aparecimento dos periódicos, observa-se que o periódico Cadernos de Terapia Ocupacional da UFSCar se destaca com 14 artigos (42,5\%), seguido da Revista de Terapia Ocupacional da USP, na qual tem 9 (27,3\%) dos artigos. Com menor frequência estão a Revista Brasileira de Educação Especial com 4 artigos (12,1\%) e a Revista Educação Especial com 3 artigos (9,1\%). A tabela 1 detalha a distribuição dos artigos nos periódicos. 
Tabela 1. Distribuição dos artigos nos periódicos.

\begin{tabular}{l|c|c}
\hline \multicolumn{1}{c|}{ Periódico } & Frequência Absoluta & Frequência Relativa (\%) \\
\hline $\begin{array}{l}\text { Cadernos de Terapia } \\
\text { Ocupacional da UFSCar }\end{array}$ & 14 & 42,5 \\
\hline $\begin{array}{l}\text { Revista de Terapia Ocupacional } \\
\text { da USP }\end{array}$ & 9 & 27,3 \\
\hline $\begin{array}{l}\text { Revista Brasileira de Educação } \\
\text { Especial }\end{array}$ & 4 & 12,1 \\
\hline Revista Educação Especial & 3 & 9,1 \\
\hline Estudos de Psicologia & 1 & 3,0 \\
\hline $\begin{array}{l}\text { Revista Brasileira em Promoção } \\
\text { de Saúde }\end{array}$ & 1 & 3,0 \\
\hline Temas sobre Desenvolvimento & 33 & 3,0 \\
\hline Total & 1 & 100 \\
\hline
\end{tabular}

Observando os periódicos, verifica-se que, dentre as publicações científicas recuperadas sobre a atuação do TO no contexto da educação inclusiva, a grande maioria se encontra em periódicos de Terapia Ocupacional em detrimento de poucas publicações em periódicos de outras áreas, como Educação Especial e Psicologia. Uma reflexão que talvez possa ser realizada, diz respeito ao público alvo da produção científica da Terapia Ocupacional na área da educação inclusiva, que são, em sua maior parte, os próprios terapeutas ocupacionais, demonstrando que esta produção ainda não é suficiente para modificar a realidade apontada por Ide, Yamamoto e Silva (2011), sobre o desconhecimento de profissionais envolvidos na educação acerca do papel do terapeuta ocupacional no contexto educacional.

Sobre os periódicos identificados na pesquisa, verifica-se também uma intersecção da área da saúde com a da educação, visto que a inclusão escolar é um processo multidisciplinar. A equipe inclusiva, segundo Schaffner e Buswel (1999) e Pelosi e Nunes (2011), pode incluir diretores, pais, professores especialistas em Educação Especial, professores de classe comum, psicólogos, terapeutas ocupacionais, supervisores, entre outros, criando-se uma rede de apoio que possa atender a diversidade de alunos da escola inclusiva, compartilhando conhecimento sobre métodos, técnicas e atividades que auxiliem professores e alunos a serem bem sucedidos em seus papéis. Essa realidade, de multidisciplinaridade envolvida nos processos de inclusão escolar, é também observada na literatura científica, sendo comum encontrar temáticas inerentes à Terapia Ocupacional e inclusão em periódicos de outras áreas.

Com relação à autoria dos artigos, os dados mostram que os 33 artigos recuperados foram escritos por um total de 63 autores, e destes apenas 10 (16\%) tem mais de um trabalho publicado sobre a temática investigada no estudo. A tabela 2 apresenta os autores com mais de um trabalho publicado. 
Aline Cirelli Coppede - Alyne Kalyane Câmara de Oliveira - Fernanda Duarte Rosa - Maria Cristina Piumbato Innocentini Hayashi

Tabela 2. Indicadores de produtividade dos autores.

\begin{tabular}{c|c|c}
\hline Autor & Frequência Absoluta & Frequência Relativa (\%) \\
\hline MARTINEZ,C.M.S. & 5 & 15 \\
\hline ASSIS, C.P. & 3 & 9 \\
\hline ROCHA, E.F. & 3 & 9 \\
\hline ARAUJO, R.C.T. & 2 & 6 \\
\hline BALEOTTI, L.R. & 2 & 6 \\
\hline LOURENCO, G.F. & 2 & 6 \\
\hline MAGALHAES, L.C. & 2 & 6 \\
\hline MANCINI, M.C. & 2 & 6 \\
\hline PALHARES, M.S. & 2 & 6 \\
\hline PELOSI, M.B. & 2 & 6
\end{tabular}

Os resultados mostram que do total de 63 autores, apenas 10 (16\%) são responsáveis por $75 \%$ da produção científica sobre essa temática, enquanto que 53 (84\%) autores representam $25 \%$ da produção científica da área. Esse achado está de acordo com uma das três leis básicas da bibliometria, a Lei de Lotka. Conforme Araújo (2006), a Lei de Lotka diz que uma grande proporção da literatura científica é produzida por um pequeno número de autores e que um grande número de pequenos produtores se iguala, em produção, ao reduzido número de grandes produtores.

Observa-se também que a maioria dos artigos, ou seja 29 (88\%), foram escritos em coautoria, isto é, com 2 a 4 autores, sendo que apenas 4 (12\%) artigos são de autoria individual. A colaboração científica entre autores, instituições e grupos de pesquisa, é justificada por alguns fatores como a Síndrome POP - Publish or Perish, cujo lema é: "publique ou pereça", forçando o pesquisador a publicar cada vez mais trabalhos científicos para alcançar um posicionamento profissional favorável; pelo suporte financeiro recebido em determinados estudos, e, também, porque as investigações estão cada vez mais complexas, especializadas e custosas, justificando maior colaboração, muitas vezes multidisciplinar (DONATO e OLIVEIRA, 2006; OLIVEIRA e GRACIO, 2008).

Quanto a tipologia dos estudos, foram encontrados 17 (52\%) artigos originais que englobam estudos transversais, descritivos e de intervenção, 12 (36\%) estudos de revisão, e 4 (12\%) artigos do tipo relato de caso. Esses dados apoiam a visão de Martinez (2005), que vincula a qualidade de um periódico ao maior número de artigos originais publicados. Dessa forma, a tendência é publicar artigos originais resultantes de pesquisas científicas significativas para a área específica do periódico. Porém, os periódicos podem incluir outros tipos de contribuições, como artigos de revisão, comunicações, resenhas e relatos de casos. Para o campo científico da Terapia Ocupacional na inclusão escolar, a pesquisa realizada verificou que os artigos originais e as revisões são os tipos que mais se destacam.

Com relação ao delineamento de pesquisa, segundo Gil (2002), refere-se ao planejamento da mesma em sua dimensão mais ampla, ou seja, o pesquisador deve 
estabelecer os meios técnicos da investigação prevendo-se os instrumentos e procedimentos necessários que utilizará para a coleta de dados. Dessa perspectiva, foram identificados 3 tipos de delineamentos nos artigos selecionados: exploratório, descritivo e experimental. A tabela 3 apresenta os resultados obtidos.

Tabela 3. Tipo de delineamento dos artigos.

\begin{tabular}{l|c|c}
\hline \multicolumn{1}{c|}{ Delineamento } & Frequência Absoluta & Frequência Relativa (\%) \\
\hline Exploratório & 15 & 46 \\
\hline Descritivo & 14 & 42 \\
\hline Experimental & 4 & 12 \\
\hline Total & 33 & 100 \\
\hline
\end{tabular}

Os resultados mostram que a maioria das pesquisas são exploratórias (46\%) e descritivas (42\%), com apenas $4(12 \%)$ pesquisas experimentais. Estes dados condizem com a definição dos delineamentos, os quais descrevem que as pesquisas exploratórias apresentam como objetivo: o aprimoramento de ideias, proporcionar maior familiaridade com o problema, com vistas a torná-lo mais explícito. Já as pesquisas descritivas têm como objetivo a descrição das características de uma determinada população, fenômeno ou estabelecer relações entre variáveis, e as pesquisas experimentais identificam os fatores que determinam ou que contribuem para a ocorrência dos fenômenos. Uma pesquisa experimental pode ser a continuação de outra descritiva, pois explicar um fenômeno exige sua descrição detalhada (GIL, 2002; MARCONI e LAKATOS, 2002; CRUZ e RIBEIRO, 2004).

No campo da inclusão escolar, é necessário, muitas vezes, primeiramente entender os fenômenos, identificar fatores de risco, como as barreiras físicas e atitudinais presentes no contexto da escola, para, posteriormente, pensar em ações e estratégias de intervenção. Desta forma, as pesquisas exploratórias e descritivas podem se configurar como sendo uma primeira etapa. Enquanto que, para realização de pesquisas experimentais é necessário prévio conhecimento e controle sobre as variáveis, exigindo, inclusive, mais tempo para a realização deste tipo de investigação. Não obstante, compreende-se a relevância de cada tipo de pesquisa, com seus objetivos específicos e contribuições complementares para o estado da arte.

Tabela 4. Indicadores das temáticas mais abordadas.

\begin{tabular}{l|c|c}
\hline \multicolumn{1}{c|}{ Temas } & Frequência Absoluta & Frequência Relativa (\%) \\
\hline $\begin{array}{l}\text { Atuação do TO na inclusão } \\
\text { escolar de pessoas com } \\
\text { necessidades educacionais } \\
\text { especiais }\end{array}$ & 10 & 30,3 \\
\hline $\begin{array}{l}\text { Discussão sobre a TO na } \\
\text { inclusão escolar }\end{array}$ & 8 & 24,2 \\
\hline $\begin{array}{l}\text { Continuação Tabela 4 } \\
\text { Atuação do TO na Tecnologia } \\
\text { Assistiva para a inclusão escolar }\end{array}$ & 4 & 12,1 \\
\hline
\end{tabular}


Aline Cirelli Coppede - Alyne Kalyane Câmara de Oliveira - Fernanda Duarte Rosa - Maria Cristina Piumbato Innocentini Hayashi

\begin{tabular}{l|c|c}
\hline $\begin{array}{l}\text { Percepção de professores sobre a } \\
\text { inclusão escolar de crianças com } \\
\text { deficiência física }\end{array}$ & 2 & 6,1 \\
\hline $\begin{array}{l}\text { Participação escolar de alunos } \\
\text { com deficiência }\end{array}$ & 2 & 6,1 \\
\hline $\begin{array}{l}\text { Discussão sobre a } \\
\text { Terapia Ocupacional na } \\
\text { educação }\end{array}$ & 1 & 3,0 \\
\hline Consultoria colaborativa & 1 & 3,0 \\
\hline $\begin{array}{l}\text { Cotidiano de crianças com } \\
\text { deficiência incluídas }\end{array}$ & 1 & 3,0 \\
\hline $\begin{array}{l}\text { Estratégias pedagógicas de } \\
\text { professores para inclusão escolar }\end{array}$ & 1 & 3,0 \\
\hline $\begin{array}{l}\text { Formação de professores para } \\
\text { inclusão escolar }\end{array}$ & 1 & 3,0 \\
\hline $\begin{array}{l}\text { Percepção de mães sobre a } \\
\text { inclusão escolar de crianças } \\
\text { com necessidades educacionais } \\
\text { especiais }\end{array}$ & 1 & 3,0 \\
\hline $\begin{array}{l}\text { Política pública de inclusão } \\
\text { escolar }\end{array}$ & 33 & 3,0 \\
\hline \multicolumn{1}{c}{ Total } & 100 \\
\hline
\end{tabular}

A tabela 4 mostra os principais temas encontrados nos artigos analisados, sendo que o mais frequente se relaciona a própria atuação do terapeuta ocupacional na inclusão escolar de pessoas com necessidades educacionais especiais (10 artigos, isto é 30,3\%). Dentre esses artigos os temas estudados abordavam Deficiência intelectual, Deficiência física, Paralisia cerebral, Mielomeningocele e Dificuldade de aprendizagem. Observa-se que, apesar dos estudos focarem a atuação da Terapia Ocupacional na educação inclusiva, eles não abrangem toda a diversidade do alunado com necessidades educacionais especiais, visto que, no ambiente da escola inclusiva, convivem simultaneamente alunos com diferentes comprometimentos (motores, intelectuais, sensoriais e comportamentais), sendo necessário criar soluções ao mesmo tempo particulares e coletivas, conforme afirmam Rocha, Luiz e Zulian (2003).

Outro tema frequente é o que abrange discussões sobre a Terapia Ocupacional na inclusão escolar, com 8 artigos identificados (24,2\%). Esses estudos apontam para as possibilidades de atuação da Terapia Ocupacional no contexto da inclusão escolar, demonstrando que há uma necessidade de se afirmar enquanto ciência e profissão quando se trata da interface com o campo educacional, evidenciando o seu papel e as diversas formas de contribuição dentro do universo particular da escola. Pois, de fato, de acordo com Munguba (2007), é preciso que o TO comece a se comunicar bem com esse universo, para então se incluir nele e fazer diferença.

Uma importante área da atuação do TO, na inclusão escolar, é a aplicação da Tecnologia Assistiva que, segundo Shuster (1993), prioriza a função para realizar tarefas específicas do cotidiano, inclusive em ambiente escolar, sendo objetivo da Terapia Ocupacional nesse contexto o uso da Tecnologia Assistiva para o aumento 
da independência do aluno com necessidades educacionais especiais. Nessa direção, justifica-se a localização de 4 artigos (12,1\%) que tratam especificamente da atuação do TO na Tecnologia Assistiva para a inclusão escolar.

Outro resultado revelado na pesquisa foi a existência de 3 artigos (9,1\% do total) sobre a percepção de professores $(6,1 \%)$ e pais (3\%) sobre a inclusão escolar. Uma possível explicação para o interesse nessa temática pode ser buscada na importância de compreender as concepções de professores e familiares, enquanto atores diretamente envolvidos no contexto da educação inclusiva, uma vez que o ponto de vista destes atores podem revelar dificuldades por eles enfrentadas e suas expectativas, além de determinar a ação deles no processo de inclusão escolar.

Demais artigos relacionados à atuação do TO, nos processos de inclusão escolar de forma indireta (atuação na educação como um todo), também foram identificados, contudo, questões de escopo político sobre a forma como este profissional pode atuar na inclusão escolar, conforme apontam Toyoda et al. (2007), e a questão da maior instrumentalização e qualificação profissional nos cursos de formação em Terapia Ocupacional para atuar de maneira mais satisfatória nos processos de inclusão escolar, segundo apontam Andrade e Braga (2009), estão sendo deixadas de lado nas produções científicas, questões estas que muito ajudariam a responder as principais dificuldades apontadas pela literatura para a inserção do TO na educação inclusiva.

\section{Considerações finais}

De uma forma geral, a análise bibliométrica apontou que a produção científica sobre a atuação do terapeuta ocupacional, na educação inclusiva, apresentou um crescimento significativo nos últimos anos, demonstrando uma maior preocupação na divulgação destas práticas e estudos. No entanto, é perceptível também que esta produção, em sua maior parte ainda se encontra restrita aos periódicos de Terapia Ocupacional, sendo poucos os trabalhos publicados em periódicos voltados aos demais campos de conhecimento e atuação profissional, como os da Educação, Educação Especial e Psicologia. Percebe-se, também, que a produção científica de Terapia Ocupacional, na área de inclusão escolar, acompanha as tendências científicas gerais, dos quais, um número reduzido de autores detém um maior número de publicações na área, com um número grande de autorias compartilhadas.

Os delineamentos mais encontrados foram os artigos originais e artigos de revisão e as temáticas são diversificadas. Apesar dos achados revelarem importantes contribuições da Terapia Ocupacional, como diferentes formas e abordagens de atuação do terapeuta ocupacional no processo de inclusão escolar de alunos com necessidades educacionais especiais, evidenciando o papel deste profissional na interface com o campo da Educação Especial, há temas, ainda, a serem explorados, como a questão política e a qualificação profissional do próprio terapeuta ocupacional para atuar em contextos de inclusão escolar, ressaltando a importância de que estes estudos sejam divulgados para os demais profissionais envolvidos na educação inclusiva, de forma a auxiliar, no conhecimento destes profissionais, sobre a existência, características e importância da atuação do terapeuta ocupacional na área. 


\section{Referências}

ANDRADE, A. F.; BRAGA, A. P. M. Percepção de profissionais da educação inclusiva acerca da terapia ocupacional. Revista Brasileira em Promoção da Saúde. v. 22, n. 3, p. 143-150, 2009.

BRASIL. MEC/INEP. Censo escolar 2005. Brasília: MEC/INEP, 2005.

ARAÚJO, C. A. Bibliometria: evolução histórica e questões atuais. Em Questão. v. 12, n. 1, p. 11-32, 2006.

CRUZ, C:; RIBEIRO, U. Metodologia Científica: teoria e pratica. Rio de Janeiro: Axcel, 2004.

DONATO, H. M.; OLIVEIRA, C. F. Patologia mamária: avaliação da atividade científica nacional através de indicadores bibliométricos. Acta Médica Portuguesa. v. 19, p. 225-234, 2006.

GIL, A. C. Como elaborar projetos de pesquisa. 4ed. São Paulo: Atlas, 2002.

HAYASHI, M. C. P. I. et al. Protocolo para coleta de dados bibliométricos em bases de dados. Mimeo. 2011.

IDE, M. G.; YAMAMOTO, B. T.; SILVA, C. C. B. Identificando possibilidades de atuação da terapia ocupacional na inclusão escolar. Cadernos de Terapia Ocupacional da UFSCar, v. 19, n. 3, p. 323-332. 2011.

LAUDELINO, J. A. S.; NAVARRO, R. M.; BEUREN, I. B. Análise da abordagem da controladoria nas dissertações e teses dos programas acadêmicos de mestrado e doutorado em ciências contábeis no Brasil. Revista de Contabilidade da UFBA, v. 4, n. 2, p. 21-33, 2010.

MARCONI, M. A.; LAKATOS, E. M. Técnicas de Pesquisa. São Paulo: Atlas, 1990.

MARTINEZ, J. A. B. Perseguindo o Medline. Jornal Brasileiro de Pneumologia, v. 31, n. 2, p. 95-96, 2005.

MUNGUBA, M. C. Inclusão escolar. In: CAVALCANTI, A.; GALVÃO,C. (Org.). Terapia ocupacional: fundamentação \& prática. Rio de Janeiro: Guanabara Koogan, 2007. p. 519-525.

OLIVEIRA, E. F. T.; GRACIO, M. C. C. Scientific collaboration network in "metrical studies": a co-authorship study using the scielo information science periodicals. Brazilian Journal of Information Science, v. 2, n. 2, p. 33-47, 2008.

PELOSI, M. B. O papel do terapeuta ocupacional na tecnologia assistiva. Cadernos de Terapia Ocupacional da UFSCar, v. 13 n. 1, p. 39-45. 2005.

PELOSI, M. B.; NUNES, L. R. D. P. A ação conjunta dos profissionais da saúde e da educação na escola inclusiva. Revista de Terapia Ocupacional da Universidade de São Paulo, v. 22, n. 1, p. 52-59, 2011.

PINHEIRO, R. C. et al. Produção científica sobre avaliação da visão em crianças: um estudo bibliométrico na base de dados LILACS. Revista de Educação Especial Santa Maria, v. 25, n. 42, p. 143-166, 2012.

PIZANNI, L.; SILVA, R. C.; HAYASHI, M. C. P. I. Base de dados e bibliometria: a presença da Educação Especial na base medline. Revista Brasileira de Biblioteconomia e Documentação, v. 4, n. 1, p. 68-85, 2008.

ROCHA, E. F.; LUIZ, A.; ZULIAN, M. A. R. Reflexões sobre as possíveis contribuições da terapia ocupacional nos processos de inclusão escolar. Revista de Terapia Ocupacional da Universidade de São Paulo, v. 14, n. 2, p. 72-78, 2003.

SCHAFFNER, C. B.; BUSWELL, B. E. Dez elementos críticos para a criação de comunidades de ensino inclusivo e eficaz. In: STAINBACK, S.; STAINBACK, W. (Orgs.). Inclusão: um guia para educadores. Tradução de M. França. Porto Alegre: Artes Médicas Sul, 1999. p. 69-87.

SILVA, M. R.; HAYASHI, C. R. M.; HAYASHI, M. C. P. I. Análise bibliométrica e cientométrica: desafios para especialistas que atuam no campo. InCID: Revista de Ciência da Informação e Documentação, v. 2, n. 1, p. 110-129, 2011.

TOYODA, C. Y. et al. O contexto multidisciplinar da prática da terapia ocupacional frente ao paradigma da inclusão escolar. Cadernos de Terapia Ocupacional da UFSCar, v. 15, n. 2, p. 121-130. 2007.

TREVISAN, J. G.; DELLA BARBA, P. C. S. Reflexões acerca da atuação do terapeuta ocupacional no processo de inclusão escolar de crianças com necessidades educacionais especiais. Cadernos de Terapia Ocu pacional da UFSCar, v. 20, n. 1, p. 89-94, 2012. 
Produção científica da Terapia Ocupacional na inclusão escolar: interface com a Educação Especial e contribuições para o campo

\section{Correspondência}

Aline Cirelli Coppede - Universidade Federal de São Carlos, Centro de Educação e Ciências Humanas, Departamento de Educação. Rod. Washington Luiz, KM235, CEP: 13565-305 - Sao Carlos, São Paulo Brasil.

E-mail: alinecirelli@gmail.com - alynekoliveira@hotmail.com - fernandaduarterosa@yahoo.com.br dmch@ufscar.br

Recebido em 20 de março de 2013

Aprovado em 12 de novembro de 2013 
